\title{
Sox 2 is involved in paclitaxel resistance of the prostate cancer cell line PC-3 via the PI3K/Akt pathway
}

\author{
DONG LI $^{1 *}$, LI-NAN ZHAO $^{1 *}$, XIU-LAN ZHENG ${ }^{2}$, PING LIN $^{1}$, FENG LIN $^{1}$, YUE LI $^{1}$, \\ HAI-FENG ZOU ${ }^{1}$, RONG-JUN CUI ${ }^{1}$, HUI CHEN $^{3}$ and XIAO-GUANG YU ${ }^{1}$ \\ ${ }^{1}$ Department of Biochemistry and Molecular Biology, Harbin Medical University; ${ }^{2}$ Department of Ultrasonography, \\ Harbin Medical University Cancer Hospital; ${ }^{3}$ Department of Urology, the Second Affiliated \\ Hospital of Harbin Medical University, Harbin, Heilongjiang 150081, P.R. China
}

Received October 29, 2013; Accepted June 5, 2014

DOI: $10.3892 / \mathrm{mmr} .2014 .2630$

\begin{abstract}
Prostate cancer is the most commonly diagnosed type of cancer and the second leading cause of cancer-associated mortality in males. The efficacy of prostate cancer chemotherapy is frequently impaired by drug resistance; however, the underlying mechanisms of this resistance remain elusive. Sex determining region Y-box 2 (Sox2) is of vital importance in the regulation of stem cell proliferation and carcinogenesis. In the present study, using MTT, clone formation, cell cycle and apoptosis assays, over-expression of Sox 2 was demonstrated to enhance the paclitaxel (Pac) resistance of the $\mathrm{PC}-3$ prostate cancer cell line, promoting cell proliferation and exhibiting an anti-apoptotic effect. Western blot analysis revealed that the phosphoinositide 3-kinase/Akt signaling pathway was activated in cells overexpressing Sox2, and by targeting cyclin E and survivin, Sox 2 promoted G1/S phase transition and prevented apoptosis under Pac treatment. The present study provided an understanding of Pac resistance in prostate cancer and may indicate novel therapeutic methods for chemoresistant prostate cancer.
\end{abstract}

\section{Introduction}

Prostate cancer is the most commonly diagnosed cancer and the second leading cause of cancer-associated mortality in males, having remained unchanged for $>20$ years in the USA (1). Androgen ablation therapy has been shown to be effective at the initial stages of prostate cancer; however, almost all patients progress to an androgen-independent stage

Correspondence to: Professor Xiao-Guang Yu, Department of Biochemistry and Molecular Biology, Harbin Medical University, 194 Xuefu Road, Harbin, Heilongjiang 150081, P.R. China

E-mail: yxg301@163.com

* Contributed equally

Key words: Sox2, paclitaxel, drug resistance, prostate cancer, phosphoinositide 3-kinase/Akt pathway or hormone-refractory prostate cancer (HRPC), which is unresponsive to hormone deprivation (2). Currently, the standard treatment of patients with HRPC is with docetaxel, a paclitaxel (Pac; also known as taxol) derivative-based chemotherapeutic (3). However, the efficiency of this therapy is frequently impaired by drug resistance, a notable cause of mortality in this type of cancer (4). Certain genes, including octamer-binding transcription factor 4 (OCT4), have been demonstrated to be of vital importance in the formation of drug-resistant cells in prostate cancer $(5,6)$. Therefore, since it is difficult to find novel drugs for chemotherapy, identifying the molecules involved in drug resistance and applying targeted methods may improve the efficacy of prostate cancer chemotherapy.

Sex determining region Y-box 2 (Sox2), a member of the SOX family of transcription factors (7-9), is critical in the self-renewal of embryonic stem cells $(10,11)$, maintenance of pluripotency, generation of induced stem cells (12-14) and apoptosis (15-17). Aberrant overexpression of Sox 2 has been reported in neural $(16)$, respiratory $(18,19)$, reproductive $(20,21)$ and digestive system tumors $(17,22)$. In gastric and colorectal cancer stem-like cells, Sox 2 enhanced tumorigenicity and chemoresistance $(23,24)$. It has been demonstrated that Sox2 promotes esophageal carcinoma growth by regulating the phosphoinositide 3-kinase (PI3K)/Akt signaling pathway, which is key in the cell survival process (25). In addition, expression of Sox 2 has been shown to be significantly increased in prostate cancer tissue compared with normal and hyperplastic tissues. As an androgen receptor-repressed gene, Sox 2 promotes the formation of HRPC (26). Thus, targeted therapy against Sox 2 may improve the efficiency of chemotherapy in patients with drug-resistant HRPC.

Pac and its derivatives are a wide class of well-known microtubule stabilizers and have been utilized as front-line chemotherapeutic agents for several types of cancer, including prostate cancer (3). However, these drugs frequently induce drug resistance. The molecular mechanism of Pac resistance has not been clarified, although a large amount of evidence has revealed that the PI3K/Akt signaling pathway is key in the formation of drug resistance in cancer via promotion of the expression of genes imperative for cell survival, consequently providing protection against apoptosis (27-30). In ovarian cancer cells, the constitutively activated PI3K/Akt signaling 
pathway conferred resistance to Pac, which was reversed by the PI3K/Akt inhibitor LY294002 (31). As a tumor suppressor gene, phosphatin and tensin homolog (PTEN) acts as a negative regulator of the $\mathrm{PI} 3 \mathrm{~K} / \mathrm{Akt}$ signaling pathway and its loss of function is associated with the progression and aggressive behavior of numerous types of cancer $(32,33)$. Regulation of the PI3K/Akt signaling pathway through PTEN has been reported to overcome sunitinib resistance in prostate cancer cells (34). For this reason, clarifying the roles of certain genes in the PI3K/Akt signaling pathway may be a rational way to approach drug-resistant cancer.

In the present study, the impact of Sox 2 on the effects of Pac treatment, which include induction of apoptosis and inhibition of cell proliferation, were investigated in a prostate cancer cell line. In addition, the underlying mechanism of the PI3K/Akt signaling pathway was analyzed. Targeted therapy against Sox 2 administered as a co-treatment with Pac may be a promising therapy in drug-resistant HRPC.

\section{Materials and methods}

Materials. Pac and LY294002 were supplied by Sigma (St. Louis, MO, USA). Sox 2 primary antibody (ab97959, rabbit, polyclonal, 1:500) was obtained from Abcam (Cambridge, MA, USA) and antibodies against Akt (9272s, rabbit, polyclonal, 1:500) and phosphorylated (p)-Akt (4058s, rabbit, monoclonal, 1:200) were purchased from Cell Signaling Technology, Inc. (Danvers, MA, USA). Antibodies against cyclin E (sc-198, rabbit, polyclonal, 1:1,000), were purchased from Santa Cruz Biotechnology, Inc. (Santa Cruz, CA, USA). Antibody to $\alpha$-tubulin (mouse, monoclonal, 1:1,000), and anti-rabbit (goat, 1:2,000) and anti-mouse (goat, 1:2,000) secondary antibodies were obtained from Beyotime Institute of Biotechnology (Haimen, China). The MTT Cell Viability Detection kit, lactate dehydrogenase (LDH) Assay kit, Annexin V-fluorescein isothiocyanate (FITC) \& propidium iodide (PI) Double Staining Apoptosis Detection kit for flow cytometry (FCM), Cell Mitochondria Isolation kit, Propidium Iodide, Caspase-3/9 Activity Assay kit and JC-1 probe were purchased from Beyotime Institute of Biotechnology.

Cell culture and transfection. PC-3 human prostate cancer cell lines were purchased from American Type Culture Collection (Manassas, VA, USA). The cells were maintained in RPMI-1640 medium supplemented with $10 \%$ fetal bovine serum (Thermo Fisher Scientific, Waltham, MA, USA) and $100 \mathrm{U} / \mathrm{ml}$ penicillin/streptomycin (Invitrogen Life Technologies, Carlsbad, CA, USA), at $37^{\circ} \mathrm{C}$ in a humidified atmosphere $(5 \%$ $\mathrm{CO}_{2} / 95 \%$ air). The human Sox2-coding-sequence was cloned into a pcDNA3.0 vector (Invitrogen Life Technologies) and termed as pcDNA3.0 Sox2; transfection was performed using Lipofectamine ${ }^{\circledR} 2000$ (Invitrogen Life Technologies). A total of $850 \mu \mathrm{g} / \mathrm{ml} \mathrm{G} 418$ (Calbiochem, San Diego, CA, USA) was applied to select the G418-resistant cells. The cells were treated with Pac or dimethyl sulfoxide (DMSO) as a vehicle (Veh) for $48 \mathrm{~h}$, and in several cases, LY294002 was added $2 \mathrm{~h}$ prior to Pac treatment.

Cell viability analysis. The empty vector-transfected (PC-3 Mock) and pcDNA3.0 Sox2-transfected (PC-3 Sox2) cells $\left(2 \times 10^{5}\right.$ cells $/ \mathrm{ml}$ in 96 -well plates) were treated with DMSO
$(1: 1,000)$ or $5 \mu \mathrm{M}$ Pac for $48 \mathrm{~h}$. Cell viability was measured using the MTT Cell Viability Detection kit, following the manufacturer's instructions. Absorbance was read at $450 \mathrm{~nm}$ on a microplate spectrophotometer (Spectra Max M3; Molecular Devices, Sunnyvale, CA, USA).

Clone formation assay. The PC-3 Mock and PC-3 Sox 2 cells were seeded at 500 cells/well in six-well culture plates and treated with DMSO $(1: 1,000)$ or $5 \mu \mathrm{M}$ Pac for $48 \mathrm{~h}$. Following 10 days of incubation, the cell colonies were stained with crystal violet, counted and images were captured by a digital camera (BioSpectrum 810 Imaging System; UVP, Upland, CA, USA) (35). The ratio of clone formation was calculated using the following equation: Rate of clone formation $(\%)=($ clone quantity/500) x 100. The relative clone formation ratio was normalized to the PC-3 Mock DMSO group.

LDH measurement. Leakage of LDH into the cell culture medium indicated cell membrane damage. The PC-3 Mock and PC-3 Sox 2 cells were exposed to DMSO $(1: 1,000)$ or $5 \mu \mathrm{M}$ Pac for $48 \mathrm{~h}$, then the culture medium was centrifuged at $250 \mathrm{~g}$ for $10 \mathrm{~min}$, and the supernatant was transferred to a 96-well culture plate to determine the quantity of LDH according to the manufacturer's instructions. The LDH activity was reported as the percentage relative to the control level (36). Absorbance was measured at $450 \mathrm{~nm}$ on the SpectraMax M3 microplate spectrophotometer (Molecular Devices, LLC, Sunnyvale, CA, USA).

Cell cycle and apoptosis analysis. A total of $1 \times 10^{6}$ cells were seeded into a $60 \mathrm{~mm}$ dish $24 \mathrm{~h}$ before treatment, then Pac $(5 \mu \mathrm{M}) /$ Veh was added for $48 \mathrm{~h}$ (in several cases, LY294002 was added $2 \mathrm{~h}$ before Pac treatment). For the cell cycle analysis, cells were harvested, fixed with $70 \%$ ethanol and stored at $4^{\circ} \mathrm{C}$ overnight, then incubated with RNase $(25 \mu \mathrm{g} / \mathrm{ml})$ at $37^{\circ} \mathrm{C}$ for $30 \mathrm{~min}$, followed by staining with PI $(50 \mu \mathrm{g} / \mathrm{ml})$ for $30 \mathrm{~min}$ in the dark. For the apoptosis analysis, Annexin V-FITC and PI staining was performed according to the manufacturer's instructions (Beyotime Institute of Biotechnology). The stained cells were counted using a FACSCalibur Flow Cytometer (BD Biosciences, Franklin Lakes, NJ, USA). All data were analyzed and visualized by FlowJo ${ }^{\circledR}$ software Ver. 7.6.1 for Windows (TreeStar, Inc., Ashland, OR, USA).

Mitochondrial membrane potential assay. The JC-1 probe was used to measure mitochondrial depolarization in the cells. Briefly, the mitochondria were separated from the cells following the indicated treatments using the Cell Mitochondria Isolation kit, were then incubated with $1 \mathrm{ml} \mathrm{JC}-1$ staining-solution $(5 \mu \mathrm{g} / \mathrm{ml})$ for $20 \mathrm{~min}$ at $37^{\circ} \mathrm{C}$ and rinsed twice with phosphate-buffered saline. The mitochondrial membrane potentials were measured using the relative quantities of dual emissions from mitochondrial JC-1 monomers or aggregates using the Spectra Max M3 microplate spectrophotometer. The excitation wavelength was set at $485 \mathrm{~nm}$. Fluorescence intensity was detected at $525 \mathrm{~nm}$ for monomers and $590 \mathrm{~nm}$ for aggregates. Mitochondrial depolarization was indicated by an increase in the $525 / 590 \mathrm{~nm}$ fluorescence intensity ratio.

Caspase-3 and - 9 activity measurement. Caspase activity was determined using the Caspase-3/9 Activity Assay kit (Beyotime 
A
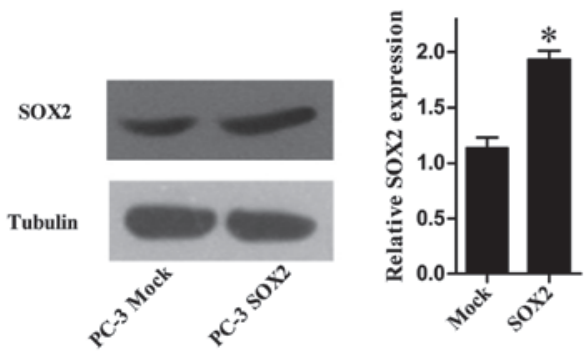

C

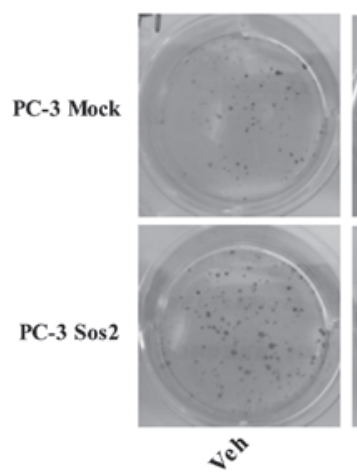

D
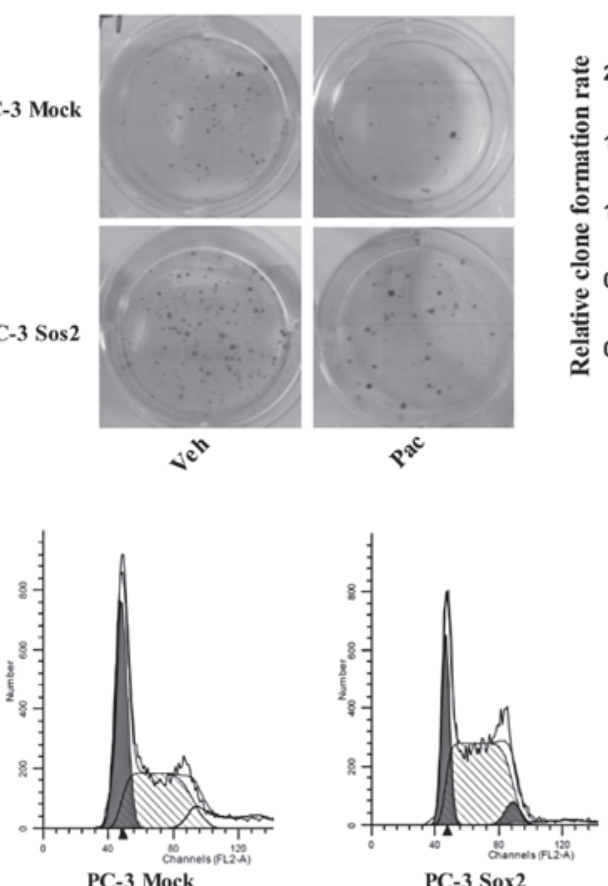

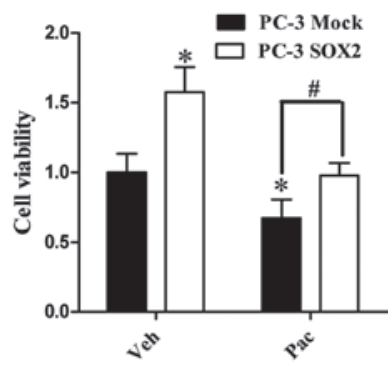

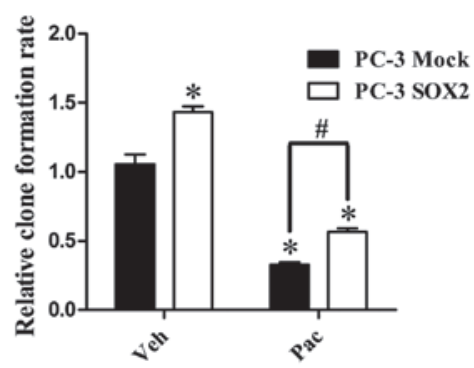

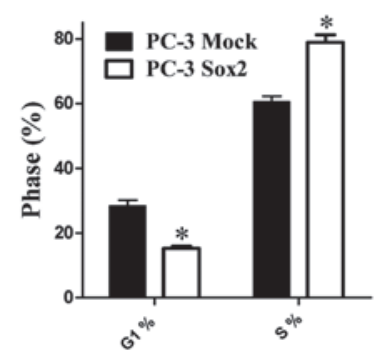

Figure 1. Sox 2 promotes cell proliferation and impairs cell cycle arrest under paclitaxel treatment in the PC-3 prostate cancer cell line. (A) Expression levels of Sox 2 in the empty vector (PC-3 Mock) and Sox 2 over-expression (PC-3 Sox2) prostate cancer cells at the protein level, normalized to $\alpha$-tubulin. (B) Cell viability of PC-3 Mock and PC-3 Sox 2 cells under Pac or Veh treatment, measured by MTT assay. (C) Colony images and clone formation rate graphs of PC-3 Mock and PC-3 Sox 2 cells with Pac or Veh treatment. (D) Cell cycle analysis of PC-3 Mock and PC-3 Sox2 under Veh, measured by flow cytometry. All data were analyzed by one-way analysis of variance and are presented as the mean \pm standard error of the mean of three independent experiments. ${ }^{*} \mathrm{P}<0.05 \mathrm{vs}$. PC-3 Mock with Veh; "P<0.05 vs. PC-3 Sox2 with Pac. Sox2, sex determining region Y-box 2; Pac, paclitaxel; Veh, vehicle (dimethylsulfoxide).

Institute of Biotechnology) following the manufacturer's instructions. The present study used 96-well microplates for incubating $10 \mu \mathrm{l}$ cell lysate in $80 \mu \mathrm{l}$ reaction buffer containing $10 \mu \mathrm{l}$ caspase substrate $(2 \mathrm{mM})$. The lysates were incubated at $37^{\circ} \mathrm{C}$ for $4 \mathrm{~h}$. Data were collected using the SpectraMax M3 microplate reader at an absorbance of $405 \mathrm{~nm}$. Caspase activity was expressed as the ratio of treated to vehicle control cells.

Transmission electron microscopy observation. The cells were fixed according to previous methods (37). The ultrastructure of the cells was examined by transmission electron microscopy (Hitachi H-600; Hitachi, Ltd., Tokyo, Japan).

Western blot analysis. Whole-cell lysate preparation and western blot analysis were conducted as described previously (38).

Statistical analysis. Each experiment was repeated in triplicate. Statistical analyses were performed using SPSS 20.0 software for Windows (IBM, Armonk, NY, USA). P $<0.05$ was considered to indicate a statistically significant difference and the results are expressed as the mean \pm the standard error of the mean.

\section{Results}

Overexpression of Sox 2 promotes cell proliferation and impairs the cell cycle arrest induced by Pac. To verify the effects of Sox 2 on PC-3 cells, cells were stably transfected with Sox 2 -expressing vector and termed PC-3 Sox2, in contrast to the empty vector PC-3 Mock cell line (Fig. 1A). To examine whether Sox 2 impacted the effect of Pac on cell proliferation, MTT and clone formation assays were performed to measure the cell proliferation status (Fig. 1B and C). The vehicle-treated PC-3 Sox 2 cells exhibited increased proliferation as compared with the mock-transfected cells, and the decreases in cell growth following Pac-treatment were significantly attenuated in PC-3 Sox 2 cells as compared with the 
A

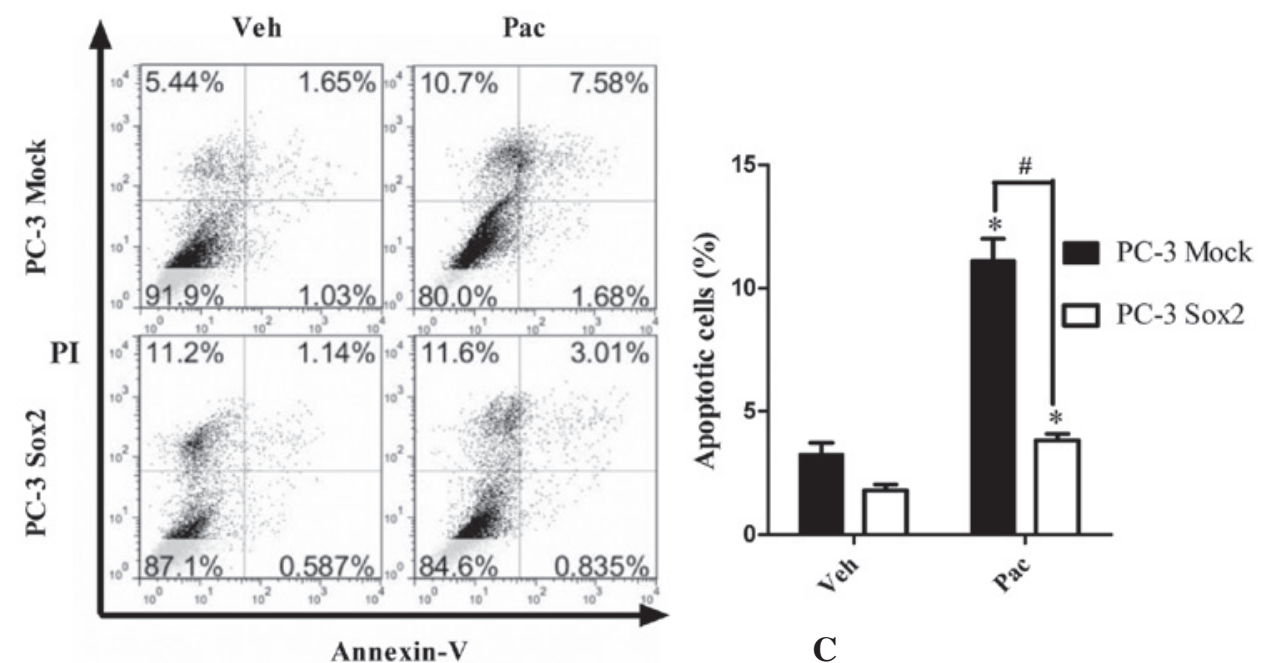

B

Annexin-V

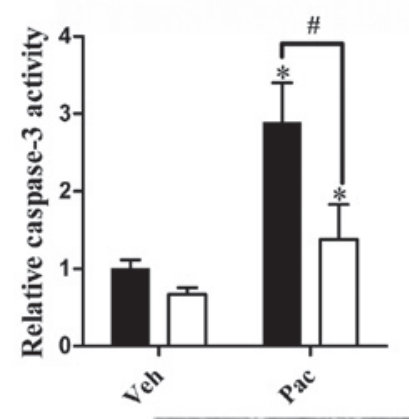

D

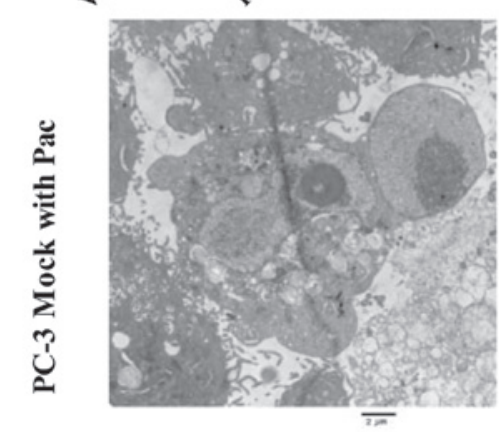

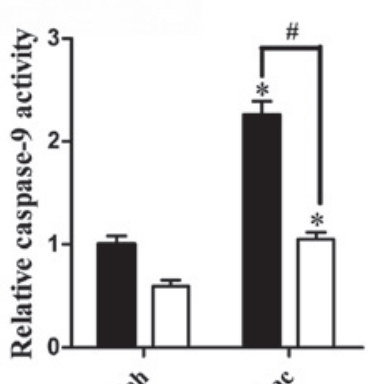

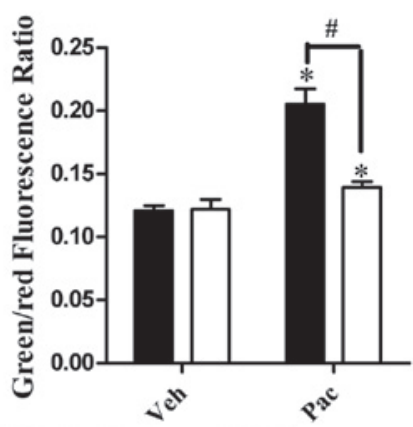

$\mathbf{E}$

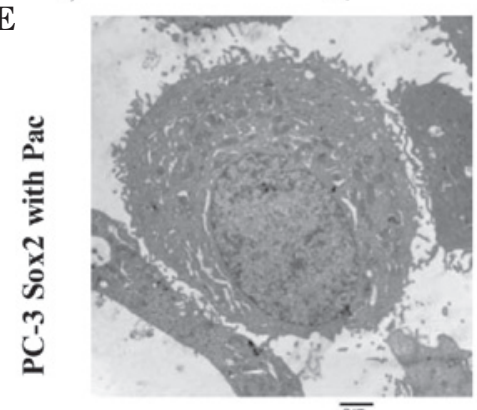

Figure 2. Sox 2 inhibits the apoptotic effect induced by Pac treatment. (A) Apoptotic cell ratio of empty vector (PC-3 Mock)/Sox2 overexpression cells (PC-3 Sox2) under Pac or Veh treatment, measured by flow cytometry-based Annexin V-fluorescein isothiocyanate and PI double staining analysis. (B) Relative activity of caspase-3, 9 in PC-3 Mock/PC-3 Sox2 cells treated with Pac or Veh. (C) Mitochondrial membrane potential of PC-3 Mock and PC-3 Sox2 cells under Pac or Veh treatment, measured by JC-1 probe. An increase in the green/red ratio indicated depolarization of the mitochondrial membrane. (D and E) Transmission electron microscopy of the morphological changes of apoptosis induced by Pac. (D) PC-3 Mock cells treated with Pac (magnification, x8,000). (E) PC-3 Sox 2 cells treated with Pac (magnification, x8,000). Results were representative of five independent experiments. All data were analyzed by one-way analysis of variance and are presented as the mean \pm standard error of the mean of three independent experiments. "P<0.05 vs. PC-3 Mock with Veh; ${ }^{\#} \mathrm{P}<0.05$ vs. PC-3 Sox 2 with Pac. PI, propidium iodide; Sox2, sex determining region Y-box 2; Pac, paclitaxel; Veh, vehicle (dimethylsulfoxide).

mock-transfected group. An FCM-based cell cycle assay was conducted to detect the cell cycle distribution of PC-3 Mock and PC-3 Sox 2 cells (Fig. 1D). The PC-3 Sox 2 cells exhibited an increased percentage of cells in S-phase as compared with the mock-transfected cells. This implies that the resistance of Sox2-overexpressing cells may be based on their upregulation of proliferation and DNA synthesis. In conclusion, the data revealed that in the Sox2-overexpressing cell line, the cell growth inhibition caused by Pac was partly attenuated.

Sox2-expression leads to evasion of apoptosis induced by Pac treatment. To examine the impact of Sox 2 on Pac-induced apoptosis, Annexin V/PI double staining and FCM analysis were used to measure the apoptotic rate of the cells (Fig. 2A). In the Mock-transfected group, the apoptotic rate was increased following incubation with Pac, which was significantly attenuated in the Sox2-overexpressing cell line. The activities of caspase- 3 and caspase- 9 increased following $48 \mathrm{~h} \mathrm{Pac}$ treatment in the PC-3 cells (Fig. 2B), which was significantly attenuated in the Sox 2-overexpressing cells, while caspase-8 activity was not significantly induced (data not shown). JC-1 aggregated in normal mitochondria and exhibited red fluorescence. Exposure of the cells to Pac for $48 \mathrm{~h}$ also resulted in dissipation of the inner mitochondrial membrane potential, which was shown as an increased green/red fluorescence ratio. By contrast to mock-transfected cells, Sox2-overexpression 
A

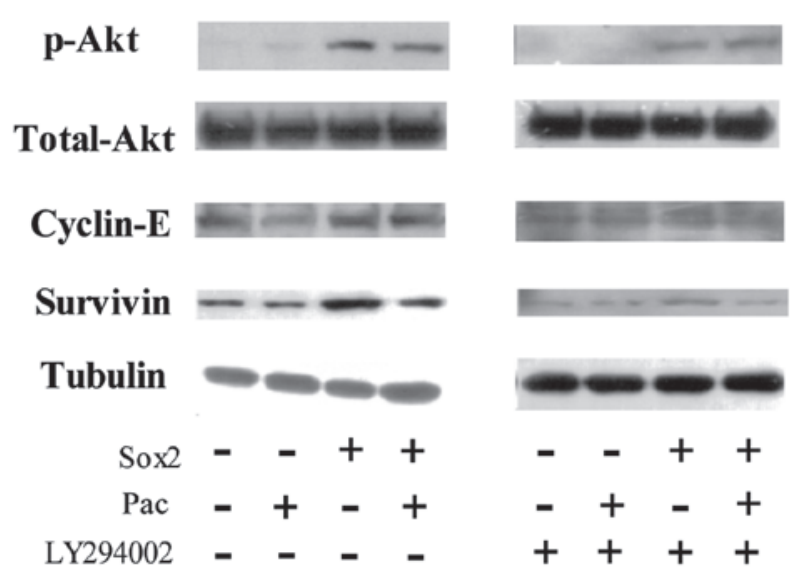

B

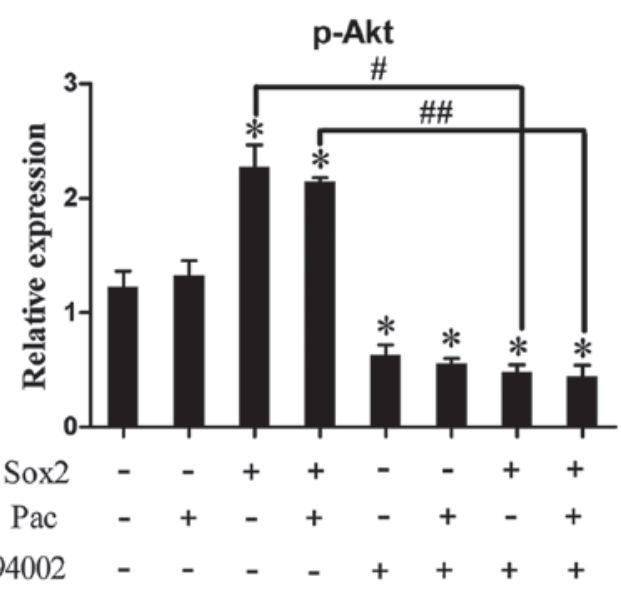

C

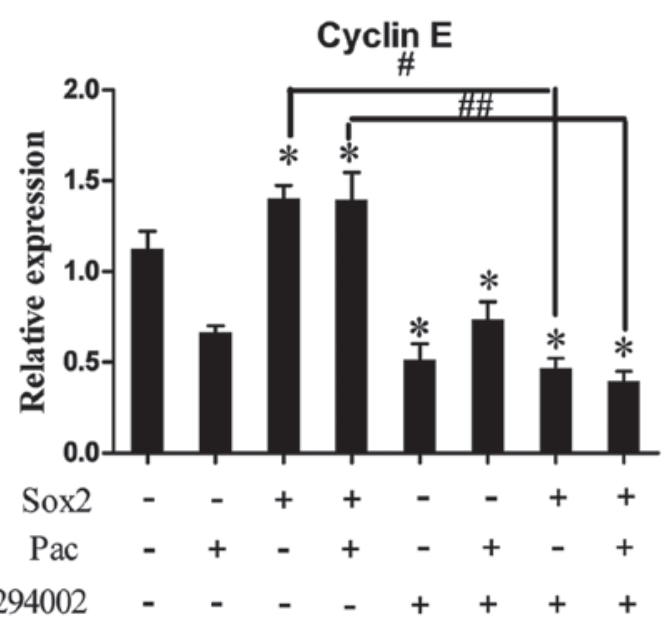

D

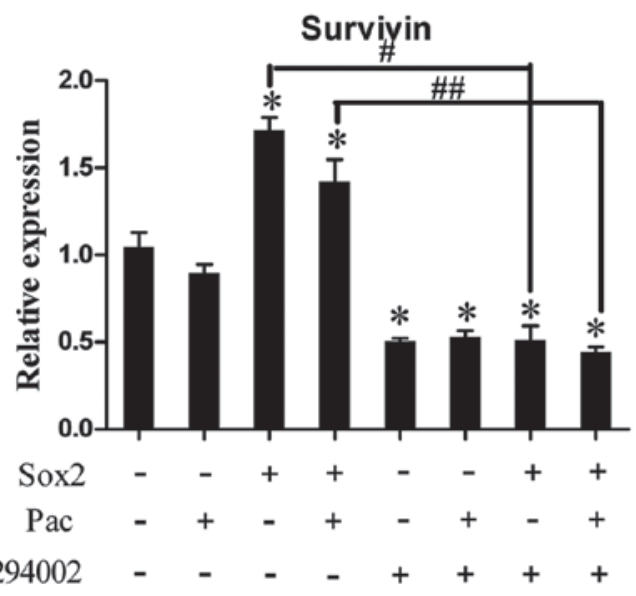

Figure 3. Expression levels of cell cycle- and apoptosis-associated proteins in PC-3 prostate cancer cell lines treated with Pac. (A) Expression levels of p-Akt, Akt, cyclin E and survivin in empty vector (PC-3 Mock; Sox 2, -) and Sox 2 overexpression (PC-3 Sox 2; Sox 2, +) cells under Pac treatment (Pac, +) or dimethyl sulfoxide (Pac, -), with or without pretreatment with the phosphoinositide 3-kinase/Akt pathway inhibitor LY294002. (B-D) Relative p-Akt, cyclin E and survivin expression levels in the different treatment groups. Data were analyzed by one-way analysis of variance and are presented as the mean \pm standard error of the mean of three independent experiments. ${ }^{*} \mathrm{P}<0.05$, vs. PC-3 Mock without Pac; ${ }^{\#} \mathrm{P}<0.05$, vs. PC-3 Sox 2 without Pac; ${ }^{\# \#} \mathrm{P}<0.05$, vs. PC-3 Sox 2 with Pac. Sox2, sex determining region Y-box 2; Pac, paclitaxel; p, phosphorylated.

completely inhibited the depolarization of the mitochondrial membrane following Pac treatment (Fig. 2C). These results indicated that Sox 2 prevented Pac-induced apoptosis. In addition, photomicrographs of PC-3 cells exposed to Pac captured by transmission electron microscopy revealed typical apoptotic morphology with chromatin condensation and formation of apoptotic bodies (Fig. 2D and E).

An LDH assay was also performed to measure the cytotoxic effect of Pac on cells; however, no significant difference was observed between the PC-3 Mock (121.4 $\pm 9.8 \%)$ and PC-3 Sox2 groups (119.7 $\pm 8.6 \%)$.

The PI3K/Akt signaling pathway is involved in the Sox2-mediated anti-apoptoticand cell proliferation-promoting effect during Pac treatment. To investigate the underlying mechanism of Sox 2 on Pac-treated cells, several signaling pathways and apoptosis- and proliferation-associated proteins were examined. p-Akt, cyclin E and survivin expression levels were found to be upregulated in PC-3 Sox 2 cells as compared with levels in mock-transfected cells (Fig. 3), while B-cell lymphoma-2 and p21 expression levels remained unchanged (data not shown). As cyclin E and survivin have been reported as Akt-regulated proteins, a PI3K inhibitor, LY294002, was used to suppress the PI3K/Akt signaling pathway activity of each group, respectively. Inhibition of the PI3K/Akt signaling pathway reduced the expression levels of $\mathrm{p}-\mathrm{Akt}$, cyclin $\mathrm{E}$ and survivin (Fig. 3), and partially attenuated the effects of Sox2 on preventing apoptosis and promoting cell proliferation under Pac treatment (Fig. 4).

\section{Discussion}

Prostate cancer is the most commonly diagnosed cancer and the second leading cause of cancer-associated mortality in males (1). In the clinic, the standard therapy for patients with HRPC is docetaxel, a Pac derivative-based chemotherapeutic. However, the efficiency of this drug is frequently impaired due to drug resistance (4). Several genes, including OCT4, have been reported to be involved in the development of drug resistance in prostate cancer cells $(5,6)$. Identifying novel molecular mechanisms underlying this drug resistance may achieve more effective chemotherapy for prostate cancer patients. 
A
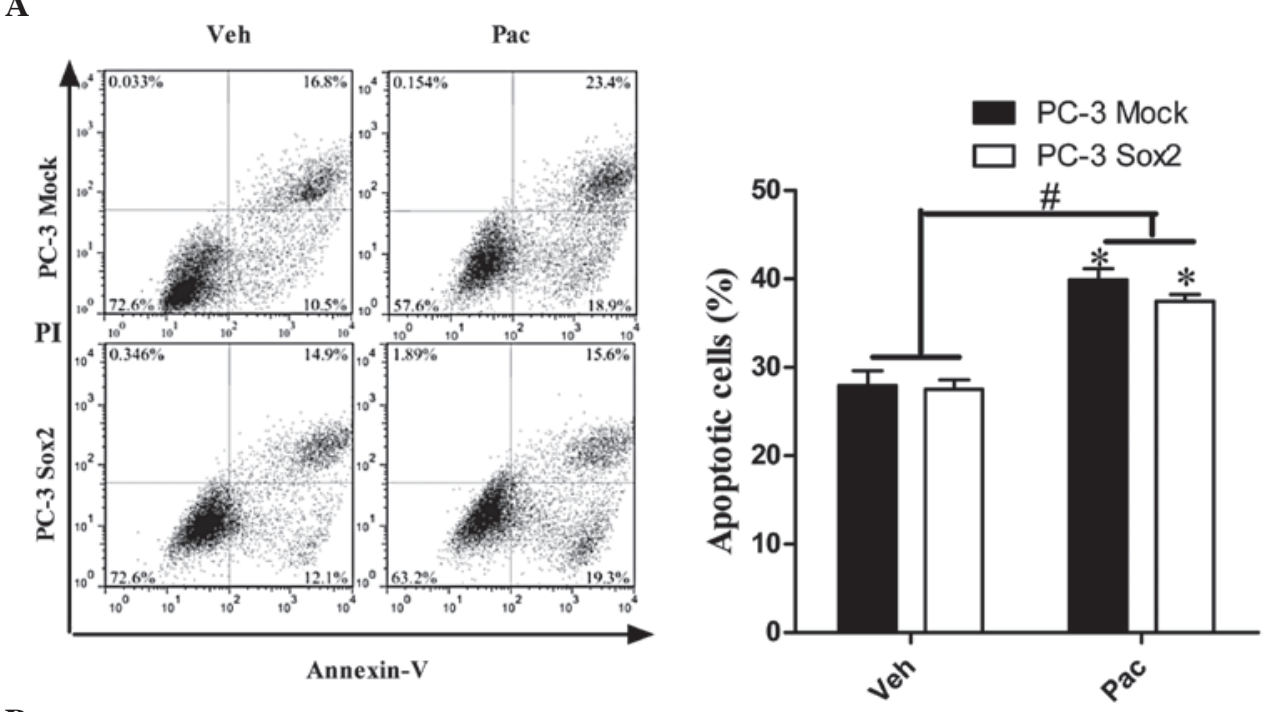

B
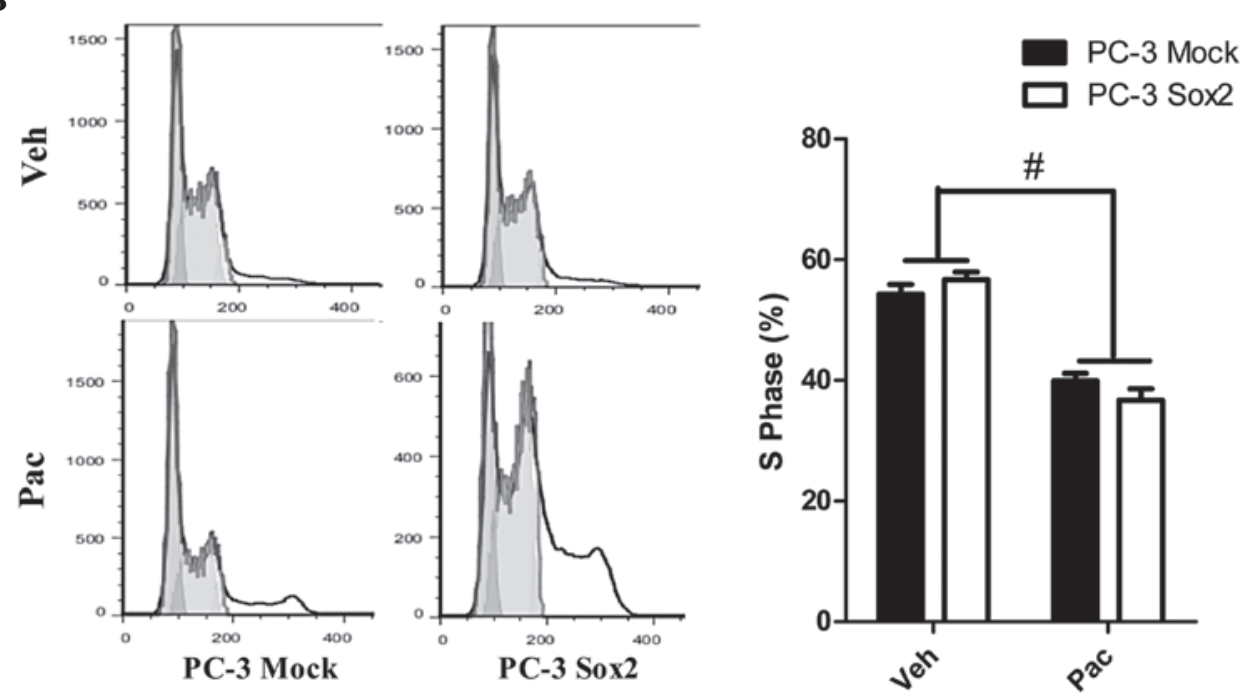

Figure 4. The PI3K/Akt pathway is involved in the Sox2-mediated anti-apoptotic and cell proliferation-promoting effects during paclitaxel treatment. (A) Apoptotic cell ratio of empty vector (PC-3 Mock)/Sox 2 over-expression (PC-3 Sox2) cells under Pac and Veh treatment, with $2 \mathrm{~h}$ pre-treatment with $30 \mu \mathrm{M}$ LY294002, measured by FCM-based Annexin V-fluorescein isothiocyanate and PI double staining analysis. (B) Cell cycle analysis of PC-3 Mock and PC-3 Sox 2 cells under Pac or Veh, with 2 h pretreatment with $30 \mu \mathrm{M}$ LY294002, measured by FCM. Data were analyzed by one-way analysis of variance and are presented as the mean \pm standard error of the mean of three independent experiments. ${ }^{~} \mathrm{P}<0.05$, vs. PC-3 Mock with Veh; ${ }^{*} \mathrm{P}<0.05$, PC-3 Mock and PC-3 Sox 2 with Pac vs. PC-3 Mock and PC-3 Sox2 with Veh. FCM, flow cytometry; PI, propidium iodide; Sox2, sex determining region Y-box 2; Pac, paclitaxel; Veh, vehicle (dimethylsulfoxide).

Pac (taxol) has been used as a chemotherapeutic agent since the 1990s. The induction of apoptosis and cell growth inhibition by Pac have been demonstrated, independent to the microtubule stabilizing effect. However, for the majority of chemotherapeutic agents, including Pac, drug resistance frequently emerges following usage. To overcome this, a higher dose may be administered; however, this inevitably induces severe cytotoxicity in normal tissues. In this regard, a therapeutic strategy involving dual agents, particularly targeted drugs, has been evaluated to reach higher therapeutic efficacy (39). The mechanism of Pac resistance is not well-characterized; however, a number of mechanisms independent to microtubule stabilization function have been suggested (40). A large body of evidence has demonstrated that the PI3K/Akt signaling pathway, which regulates a series of cell survival- and proliferation-associated genes, is key in the development of chemoresistance (27-30).
Sox 2, an important component of the 'induced pluripotent stem cell cocktail', is a member of the SOX family of transcription factors, and is critical in self-renewal of embryonic stem cells, maintenance of pluripotency, generation of induced stem cells and apoptosis. Aberrant over-expression of Sox 2 has been reported in several types of tumor (16-22). Expression of Sox2 was found to be significantly increased in the prostate cancer tissues compared with normal and hyperplastic tissues (41). Sox 2 is an androgen receptor-repressed gene and promotes the formation of HRPC. In a previous study, Sox 2 was shown to be involved in transforming growth factor- $\alpha$-induced cell proliferation and exhibit an anti-apoptotic effect in prostate cancer cells, mediated by cyclin E, p27 and survivin (38). In the present study, the results revealed that Sox 2 serves as a 'safe guard' for maintaining cell proliferation, which characterizes tumor cells. In both the PC-3 Mock and PC-3 Sox2 
cell groups, Pac inhibited cell growth, but the MTT and clone formation assay results revealed that, following Pac treatment, overexpression of Sox 2 in the PC-3 Sox 2 cells significantly promoted cell growth in comparison with the PC-3 Mock group. Furthermore, the FCM cell cycle assay indicated that overexpression of Sox 2 increased the percentage of cells in $\mathrm{S}$ phase, which suggested that Sox 2 promoted G1 to $\mathrm{S}$ phase progression. The G1/S checkpoint protein regulated by Sox 2 was thus analyzed. In accordance with previous studies (38), the expression levels of cyclin E, which combines with cyclin-dependent kinase 2 and is essential for DNA replication, and G1/S transition were examined (42). The results revealed that cyclin E expression was upregulated in PC-3 Sox 2 cells, which explains the cell cycle-promoting effects of Sox 2 .

In addition, the apoptosis-inducing effect of Pac was inhibited by Sox 2 overexpression, as measured by an FCM apoptosis assay. To verify that this decrease in the apoptotic rate was not due to decreases in necrosis, the $\mathrm{LDH}$ assay was also performed, and the data did not reveal significant differences between the PC-3 Mock and Sox 2 groups. This finding indicated that Sox 2 affected the apoptosis-inducing effect of $\mathrm{Pac}$, but not cell necrosis; and that at this concentration of Pac $(5 \mu \mathrm{M})$, necrosis induced by Pac was not evident.

By contrast to the PC-3 Mock cells, the phosphorylation levels of Akt were found to be significantly upregulated in the PC-3 Sox 2 cells, which suggested that the cell proliferation and anti-apoptotic effects of Sox 2 following Pac treatment may be mediated by activation of the PI3K/Akt signaling pathway. Survivin, a member of the inhibitor of the apoptosis-mediating protein family, is capable of regulating cell proliferation and apoptosis (43), and has been proven to be downstream of the PI3K/Akt signaling pathway (44). In the present study, survivin expression levels were detected at the protein level, and results indicated that the expression levels of survivin were significantly increased in PC-3 Sox 2 cells, which may account for the anti-apoptotic effect of Sox2.

To confirm the impact of Akt activation on cell behavior, the PC-3 cells were pre-treated with LY294002, a PI3K/Akt inhibitor. The results revealed that LY294002 inhibited the upregulation of cyclin E and survivin as well as the phosphorylation of Akt induced by Sox 2 over-expression. In addition, LY294002 inhibited the anti-apoptotic and G1/S transition promoting effects of Sox 2 following Pac treatment. All results support the hypothesis that the effects of Sox 2 are mediated by the PI3K/Akt signaling pathway. Notably, as determined by FCM, no significant differences in the percentage of apoptotic cells or the percentage of cells in $\mathrm{S}$ phase were identified between PC-3 Mock and PC-3 Sox 2 cells receiving the same treatment (Veh or Pac), which indicated that LY294002 completely inhibited the effects of Sox2. However, markedly different S-phase distribution and apoptotic percentages were observed between Veh and Pac treatment in the same cell types (PC-3 Mock or PC-3 Sox2). This suggested that Pac impacted the cell cycle distribution and cell apoptosis in multiple ways.

In conclusion, the present study indicated that: (i) Overexpression of Sox 2 exerted a drug-resistance function in PC-3 cells and may antagonize the effects of Pac; (ii) the Pac-resistance effect of Sox 2 is mediated by continuous activation of the PI3K/Akt signaling pathway; and (iii) under Pac treatment, the PI3K/Akt signaling pathway promotes cell proliferation and antagonizes apoptosis via targeting cyclin $\mathrm{E}$ and survivin. These results may indicate novel therapeutic methods for chemoresistant prostate cancer.

\section{Acknowledgements}

This study was supported by the National Youth Natural Science Foundation of China (grant no. 81101942) and the Natural Science Foundation of Education Department, Heilongjiang, China (grant no. 12531300).

\section{References}

1. Siegel R, Naishadham D and Jemal A: Cancer statistics, 2013. CA Cancer J Clin 63: 11-30, 2013.

2. Moon C, Park JC, Chae YK, Yun JH and Kim S: Current status of experimental therapeutics for prostate cancer. Cancer Lett 266: 116-134, 2008.

3. Petrylak DP: Docetaxel (Taxotere) in hormone-refractory prostate cancer. Semin Oncol 27: 24-29, 2000.

4. Seruga B, Ocana A and Tannock IF: Drug resistance in metastatic castration-resistant prostate cancer. Nat Rev Clin Oncol 8: 12-23, 2011.

5. Linn DE, Yang X, Sun F, et al: A Role for OCT4 in Tumor Initiation of Drug-Resistant Prostate Cancer Cells. Genes Cancer 1: 908-916, 2010.

6. Wu K, Xie D, Zou Y, et al: The mechanism of DAB2IP in chemo-resistance of prostate cancer cells. Clin Cancer Res 19: 4740-4749, 2013.

7. Pevny LH and Lovell-Badge R: Sox genes find their feet. Curr Opin Genet Dev 7: 338-344, 1997.

8. Wegner M: From head to toes: the multiple facets of Sox proteins. Nucleic Acids Res 27: 1409-1420, 1999.

9. Kamachi Y, Uchikawa M and Kondoh H: Pairing SOX off: with partners in the regulation of embryonic development. Trends Genet 16: $182-187,2000$.

10. Masui S, Nakatake Y, Toyooka Y, et al: Pluripotency governed by Sox 2 via regulation of Oct $3 / 4$ expression in mouse embryonic stem cells. Nat Cell Biol 9: 625-635, 2007.

11. Fong H, Hohenstein KA and Donovan PJ: Regulation of self-renewal and pluripotency by Sox 2 in human embryonic stem cells. Stem Cells 26: 1931-1938, 2008

12. Takahashi K, Tanabe K, Ohnuki M, et al: Induction of pluripotent stem cells from adult human fibroblasts by defined factors. Cell 131: 861-872, 2007.

13. Takahashi K and Yamanaka S: Induction of pluripotent stem cells from mouse embryonic and adult fibroblast cultures by defined factors. Cell 126: 663-676, 2006.

14. Yamanaka S: Strategies and new developments in the generation of patient-specific pluripotent stem cells. Cell Stem Cell 1:39-49, 2007.

15. Ben-Porath I, Thomson MW, Carey VJ, et al: An embryonic stem cell-like gene expression signature in poorly differentiated aggressive human tumors. Nat Genet 40: 499-507, 2008.

16. Gangemi RM, Griffero F, Marubbi D, et al: Sox 2 silencing in glioblastoma tumor-initiating cells causes stop of proliferation and loss of tumorigenicity. Stem Cells 27: 40-48, 2009.

17. Bass AJ, Watanabe $\mathrm{H}$, Mermel $\mathrm{CH}$, et al: Sox 2 is an amplified lineage-survival oncogene in lung and esophageal squamous cell carcinomas. Nat Genet 41: 1238-1242, 2009.

18. Güre AO, Stockert E, Scanlan MJ, et al: Serological identification of embryonic neural proteins as highly immunogenic tumor antigens in small cell lung cancer. Proc Natl Acad Sci USA 97: 4198-4203, 2000.

19. Hussenet T, Dali S, Exinger J, et al: SOX2 is an oncogene activated by recurrent 3q26.3 amplifications in human lung squamous cell carcinomas. PLoS One 5: e8960, 2010.

20. Ye F, Li Y, Hu Y, Zhou C, Hu Y and Chen H: Expression of Sox 2 in human ovarian epithelial carcinoma. J Cancer Res Clin Oncol 137: 131-137, 2011.

21. Jia X, Li X, Xu Y, et al: SOX2 promotes tumorigenesis and increases the anti-apoptotic property of human prostate cancer cell. J Mol Cell Biol 3: 230-238, 2011.

22. Saigusa S, Tanaka K, Toiyama Y, et al: Correlation of CD133, OCT4, and SOX2 in rectal cancer and their association with distant recurrence after chemoradiotherapy. Ann Surg Oncol 16: 3488-3498, 2009. 
23. Rao GH, Liu HM, Li BW, et al: Establishment of a human colorectal cancer cell line P6C with stem cell properties and resistance to chemotherapeutic drugs. Acta Pharmacol Sin 34: 793-804, 2013

24. Tian T, Zhang Y, Wang S, Zhou J and Xu S: Sox2 enhances the tumorigenicity and chemoresistance of cancer stem-like cells derived from gastric cancer. J Biomed Res 26: 336-345, 2012.

25. Gen Y, Yasui K, Nishikawa T and Yoshikawa T: SOX2 promotes tumor growth of esophageal squamous cell carcinoma through the AKT/mammalian target of rapamycin complex 1 signaling pathway. Cancer Sci 104: 810-816, 2013.

26. Kregel S, Kiriluk KJ, Rosen AM, et al: Sox 2 is an androgen receptor-repressed gene that promotes castration-resistant prostate cancer. PLoS One 8: e53701, 2013.

27. Kim SH, Juhnn YS and Song YS: Akt involvement in paclitaxel chemoresistance of human ovarian cancer cells. Ann N Y Acad Sci 1095: 82-89, 2007.

28. Fraser M, Leung B, Jahani-Asl A, Yan X, Thompson WE and Tsang BK: Chemoresistance in human ovarian cancer: the role of apoptotic regulators. Reprod Biol Endocrinol 1: 66, 2003.

29. Smith DA, Kiba A, Zong Y and Witte ON: Interleukin-6 and oncostatin-M synergize with the PI3K/AKT pathway to promote aggressive prostate malignancy in mouse and human tissues. Mol Cancer Res 11: 1159-1165, 2013.

30. Barile E, De SK, Feng Y, et al: Synthesis and SAR studies of dual AKT/NF-kappaB inhibitors against melanoma. Chem Biol Drug Des 82: 520-533, 2013.

31. Hu L, Hofmann J, Lu Y, Mills GB and Jaffe RB: Inhibition of phosphatidylinositol 3'-kinase increases efficacy of paclitaxel in in vitro and in vivo ovarian cancer models. Cancer Res 62 : 1087-1092, 2002.

32. Alimonti A, Carracedo A, Clohessy JG, et al: Subtle variations in Pten dose determine cancer susceptibility. Nat Genet 42 : 454-458, 2010

33. Trotman LC, Niki M, Dotan ZA, et al: Pten dose dictates cancer progression in the prostate. PLoS Biol 1: E59, 2003.
34. Makhov PB, Golovine K, Kutikov A, et al: Modulation of $\mathrm{Akt} / \mathrm{mTOR}$ signaling overcomes sunitinib resistance in renal and prostate cancer cells. Mol Cancer Ther 11: 1510-1517, 2012.

35. Franken NA, Rodermond HM, Stap J, Haveman J and van Bree C: Clonogenic assay of cells in vitro. Nat Protoc 1: 2315-2319, 2006.

36. Huang CC, Aronstam RS, Chen DR and Huang YW: Oxidative stress, calcium homeostasis, and altered gene expression in human lung epithelial cells exposed to $\mathrm{ZnO}$ nanoparticles. Toxicol In Vitro 24: 45-55, 2010.

37. Jiang S, Zu Y, Fu Y, Zhang Y and Efferth T: Activation of the mitochondria-driven pathway of apoptosis in human PC-3 prostate cancer cells by a novel hydrophilic paclitaxel derivative, 7-xylosyl-10-deacetylpaclitaxel. Int J Oncol 33: 103-111, 2008.

38. Lin F, Lin P, Zhao D, et al: Sox 2 targets cyclinE, p27 and survivin to regulate androgen-independent human prostate cancer cell proliferation and apoptosis. Cell Prolif 45: 207-216, 2012.

39. Sandler A, Gray R, Perry MC, et al: Paclitaxel-carboplatin alone or with bevacizumab for non-small-cell lung cancer. N Engl J Med 355: 2542-2550, 2006.

40. Yang YI, Lee KT, Park HJ, et al: Tectorigenin sensitizes paclitaxel-resistant human ovarian cancer cells through downregulation of the Akt and $\mathrm{NF \kappa B}$ pathway. Carcinogenesis 33: 2488-2498, 2012

41. Bae KM, Su Z, Frye C, et al: Expression of pluripotent stem cell reprogramming factors by prostate tumor initiating cells. J Urol 183: 2045-2053, 2010.

42. Sherr CJ: G1 phase progression: cycling on cue. Cell 79: 551-555, 1994.

43. Altieri DC and Marchisio PC: Survivin apoptosis: an interloper between cell death and cell proliferation in cancer. Lab Invest 79: 1327-1333, 1999.

44. Peng XH, Karna P, Cao Z, Jiang BH, Zhou M and Yang L: Cross-talk between epidermal growth factor receptor and hypoxia-inducible factor-1alpha signal pathways increases resistance to apoptosis by up-regulating survivin gene expression. J Biol Chem 281: 25903-25914, 2006. 